Supplementary Materials

\title{
Dynamic Photonic Barcodes for Molecular Detection Based on Cavity- Enhanced Energy Transfer
}

\author{
Yunke Zhou, ${ }^{\text {a } Z h i y i ~ Y u a n, ~}{ }^{\text {a }}$ Xuerui Gong, ${ }^{\mathrm{a}}$ Muhammad D Birowosuto, ${ }^{\text {a }}$ Cuong Dang, ${ }^{\mathrm{a}}$ \\ Yu-Cheng Chen ${ }^{\mathrm{a}, \mathrm{b}} *$
}

\begin{abstract}
a School of Electrical and Electronic Engineering, Nanyang Technological University, 50 Nanyang Avenue, 639798, Singapore

b School of Chemical and Biomedical Engineering, Nanyang Technological University, 62 Nanyang Drive, 637459, Singapore
\end{abstract}

*Correspondence Email: yucchen@ntu.edu.sq 
a

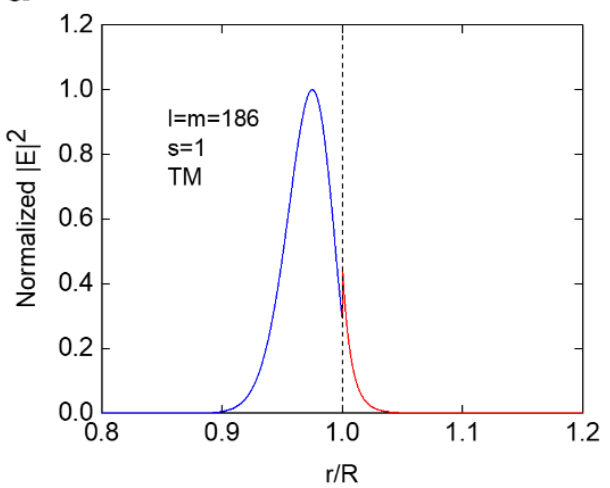

C

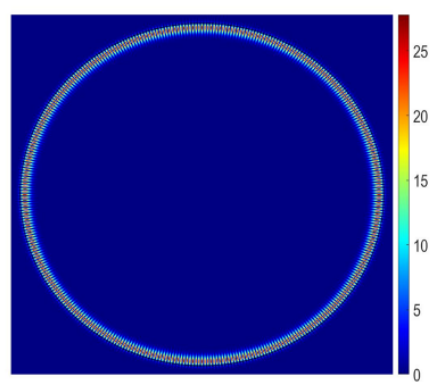

d

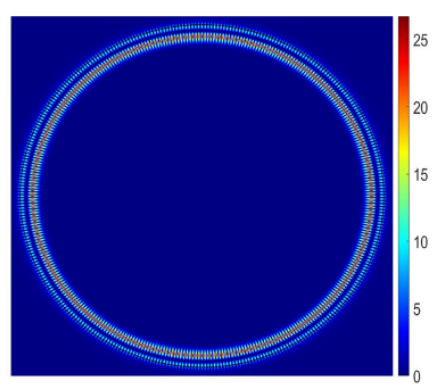

b
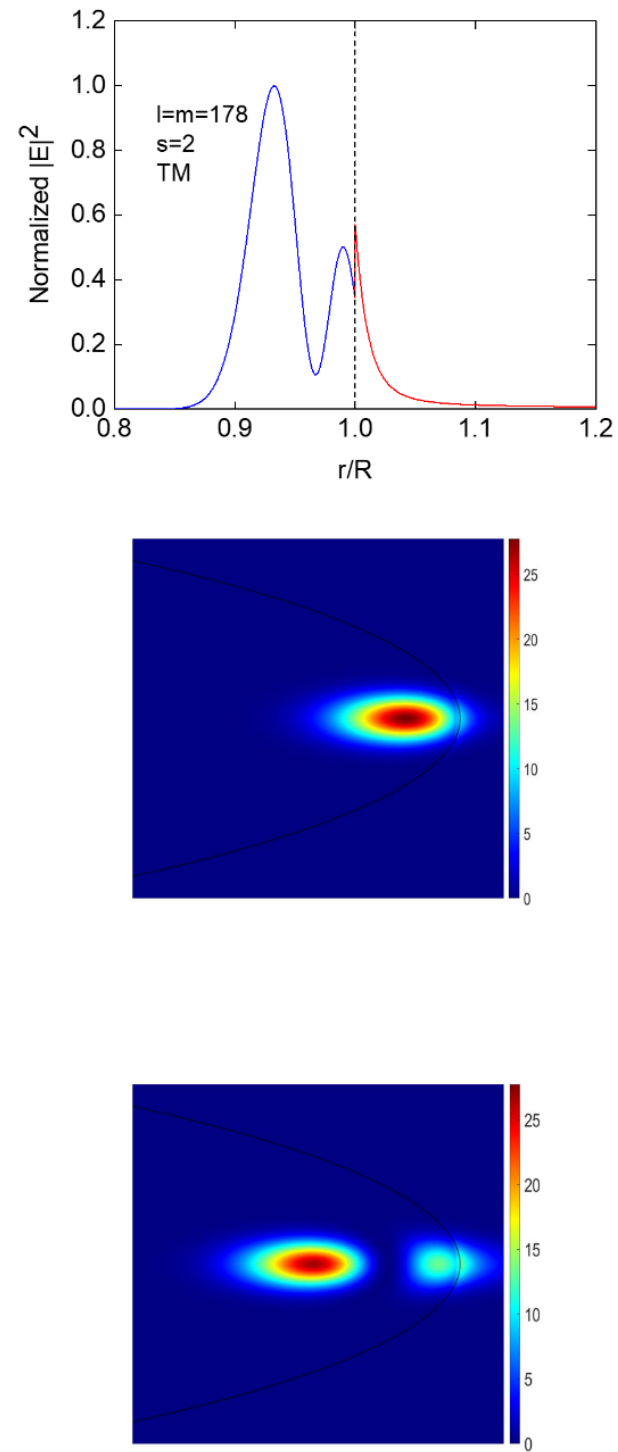

Figure S1. (a-b) Simulated results of typical radial distribution of $\left|\mathrm{E}_{\mathrm{TM}}\right|^{2}$ for an anisotropic NLC (radial) microcavity with principal diameter $=20 \mu \mathrm{m}$ and eigenwavelength $\approx 550 \mathrm{~nm}$ for radial mode numbers (a) $s=1$ and (b) $s=2$. The vertical dashed line represents the interface between the cavity and the external environment. The evanescent field is described by the red curve. (c-d) Simulated WGM hot spot distribution in the $r-\varphi$ plane (left panel) and $r-\theta$ plane (right panel) with the same parameter corresponding to (a) and (b), respectively. The edge of the cavity is shown by a black curve. 

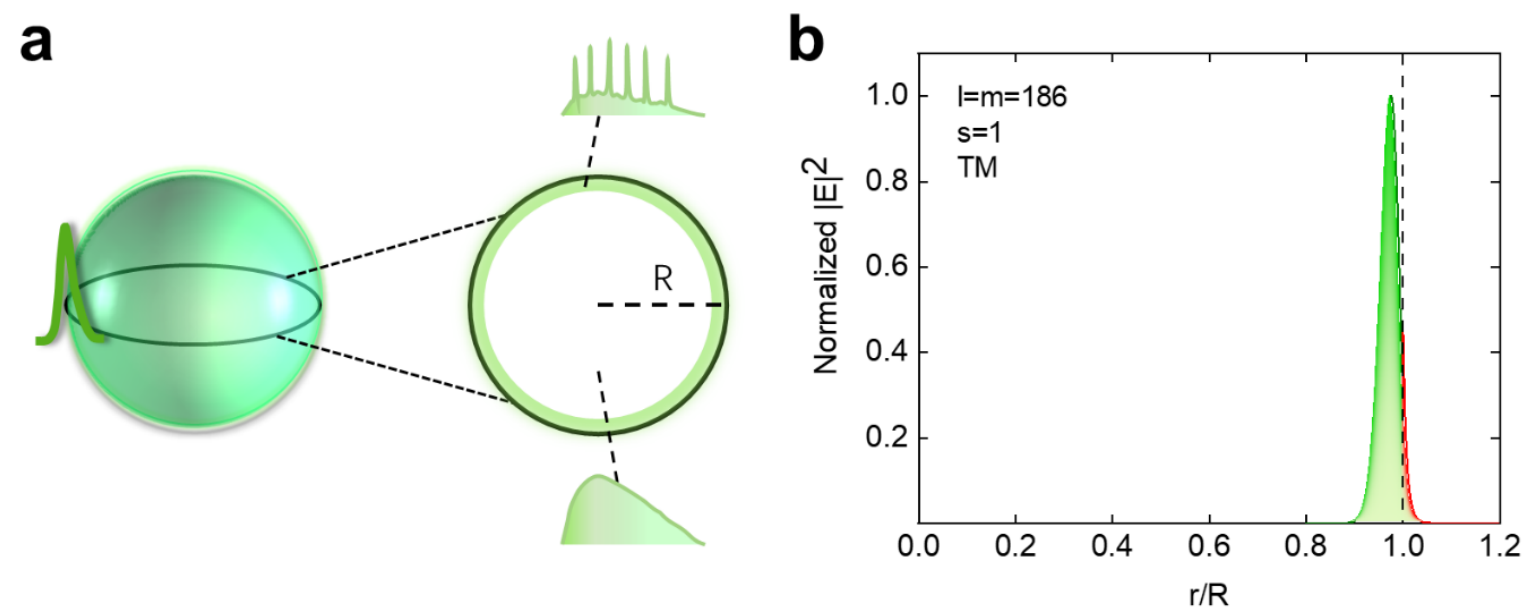

Figure S2. (a) Schematic diagram illustrating the composition of the emission signal and the corresponding region upon free space excitation and collection. Only very little proportion of the donor-acceptor molecules at the cavity interface can contribute to the WGM emission. (b) A simulated WGM distribution in the radial direction assisting the comprehension in (a). 


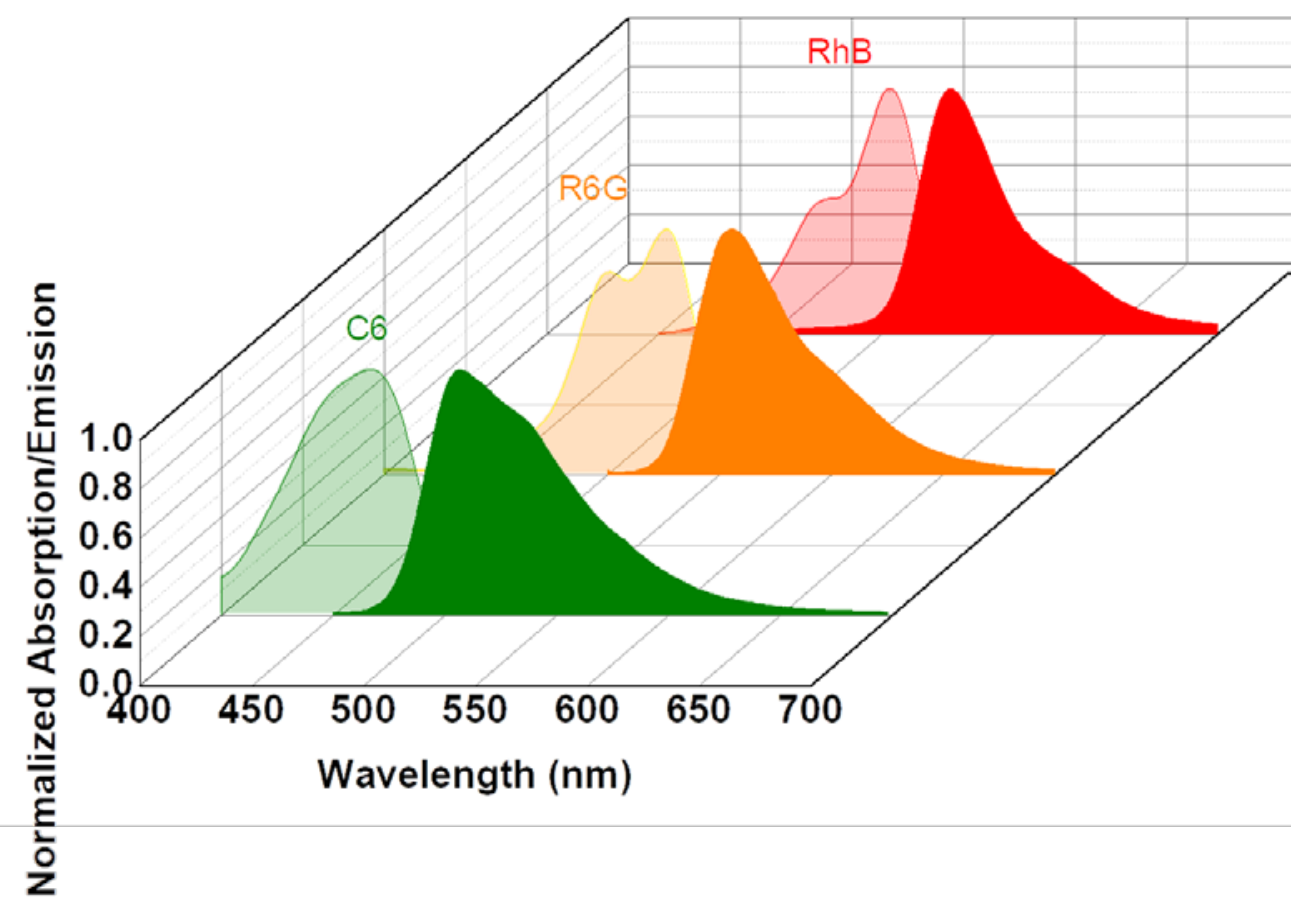

Figure S3. Normalized excitation and emission spectra of Coumarin 6 (C6): donor, Rhodamine 6G (R6G): mediator, and Rhodamine B (RhB): acceptor. 


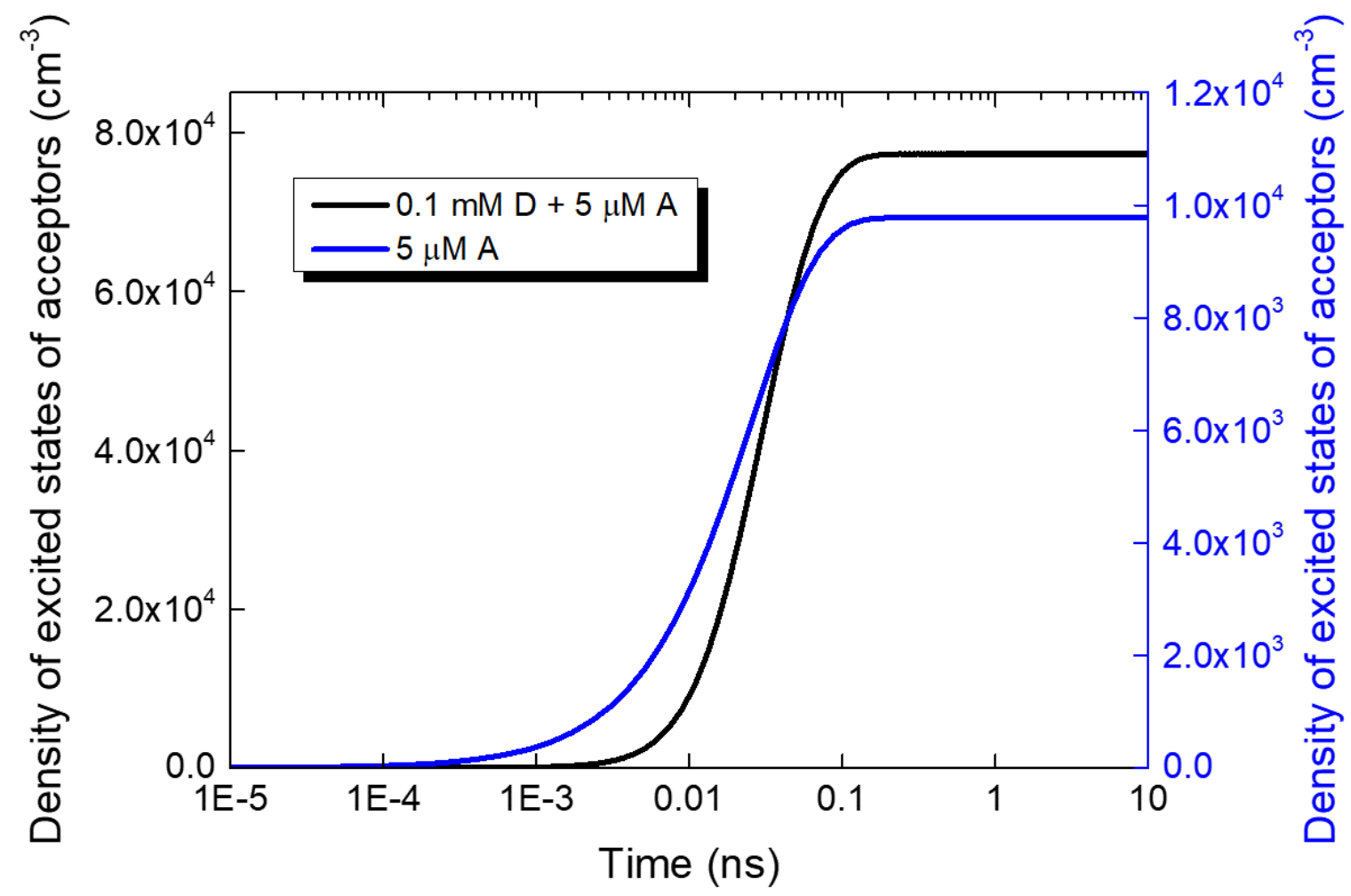

Figure S4. The simulated density of the excited states of the acceptor with $0.1 \mathrm{mM}$ donor (black curve) and without donor (blue curve) as a function of pumping time. The excitation wavelength is $460 \mathrm{~nm}$ in the presence of the donor, and $550 \mathrm{~nm}$ in the absence of the donor. The $\mathrm{x}$-axis takes the logarithm to facilitate the observation. 
a
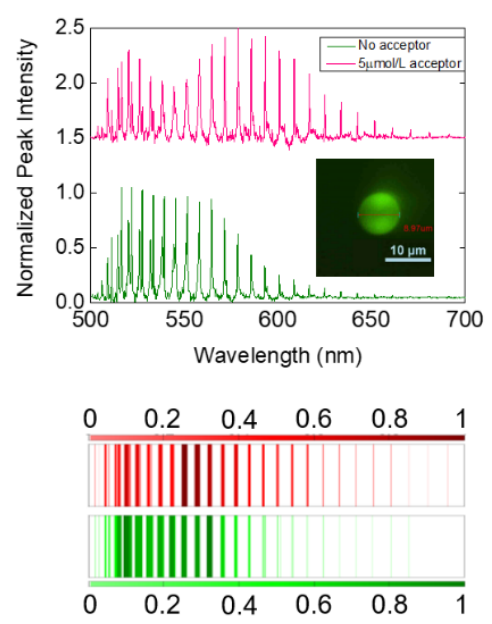

b
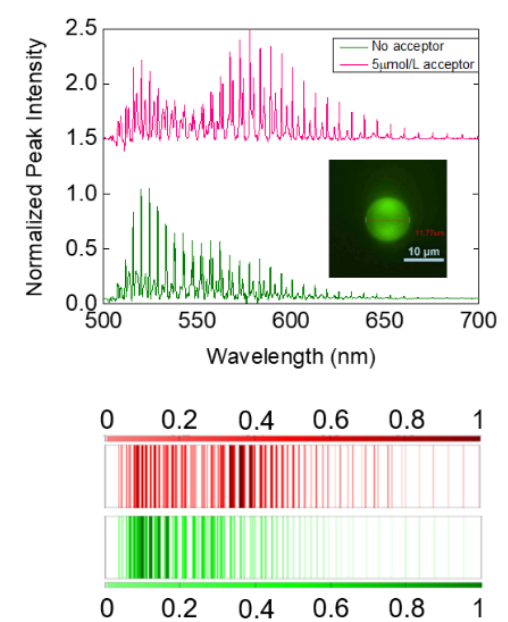

C
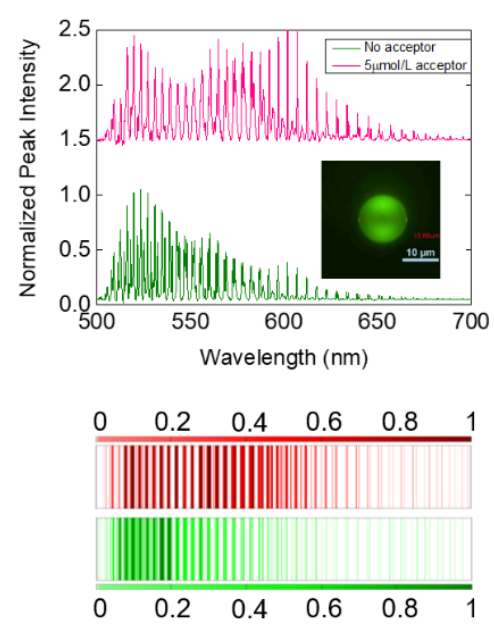

Figure S5. WGM spectra and photonic barcodes of Coumarin 6 microdroplet with a diameter (a) $8.97 \mu \mathrm{m}$, (b) $11.77 \mu \mathrm{m}$ or (c) $13.66 \mu \mathrm{m}$ before (green curve) and after (pink curve) adding $5 \mu \mathrm{M}$ Rhodamine solution. The insets present the fluorescence images captured by a monochromatic CCD (pseudocolor). All scale bars, $10 \mu \mathrm{m}$. All spectra were measured under the same excitation wavelength: 430-490 nm. The fluorescence background was subtracted for clarity. 

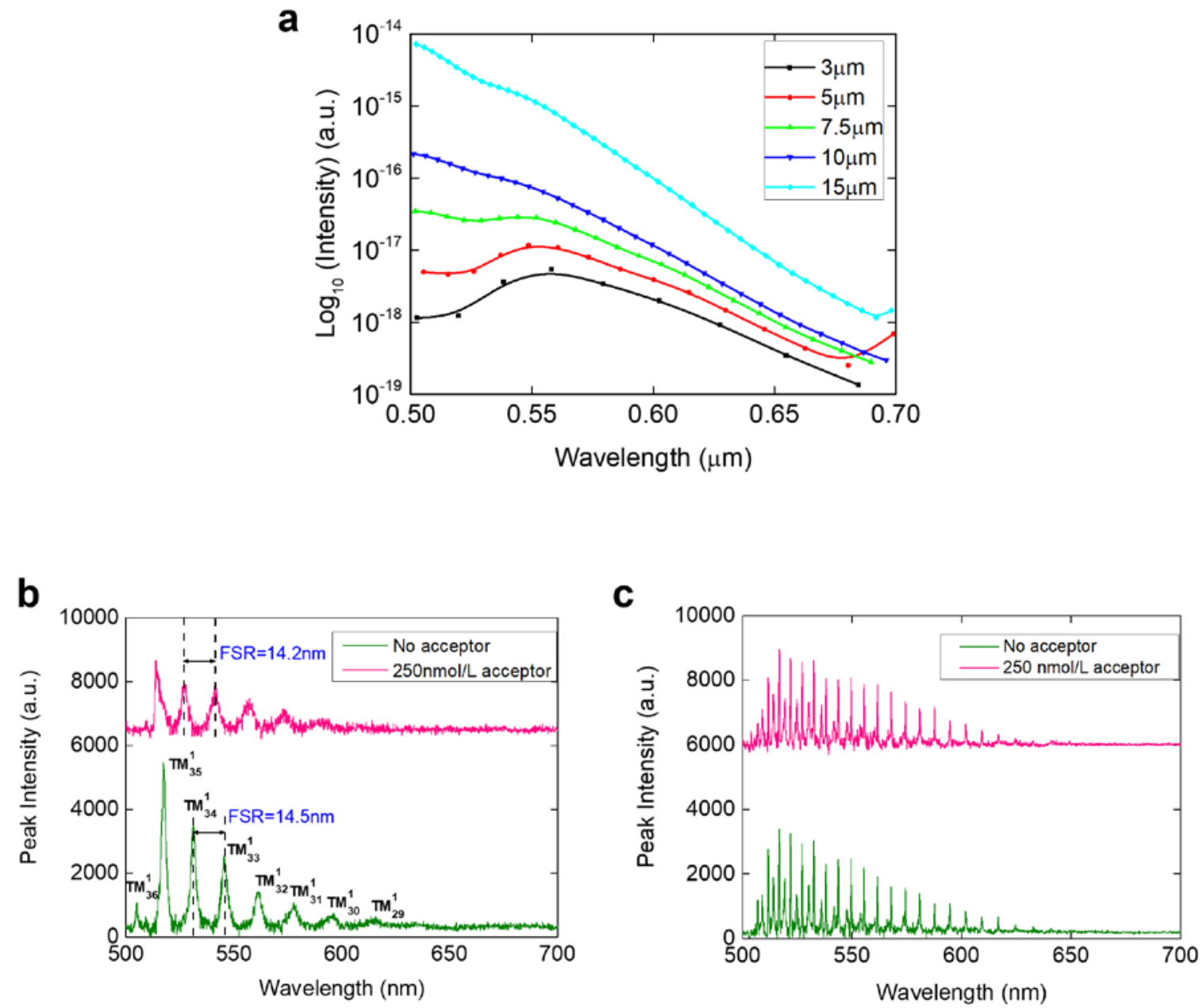

Figure S6. (a) Optical simulation of the intensity of resonant modes (the dots) under different cavity size ( $3 \mu \mathrm{m} \sim 15 \mu \mathrm{m}$ ). The acceptor concentration was fixed as a constant. The y-axis takes the logarithm to facilitate the observation. (b-c) Comparison of WGM modulated fluorescence emission spectra of Coumarin $6(50 \mu \mathrm{M})$ donor-droplet between (b) small size and (c) large size after adding low concentration of Rhodamine molecules $(250 \mathrm{nM})$. All spectra were measured under the same excitation wavelength and power density. The fluorescence background was subtracted for clarity. 


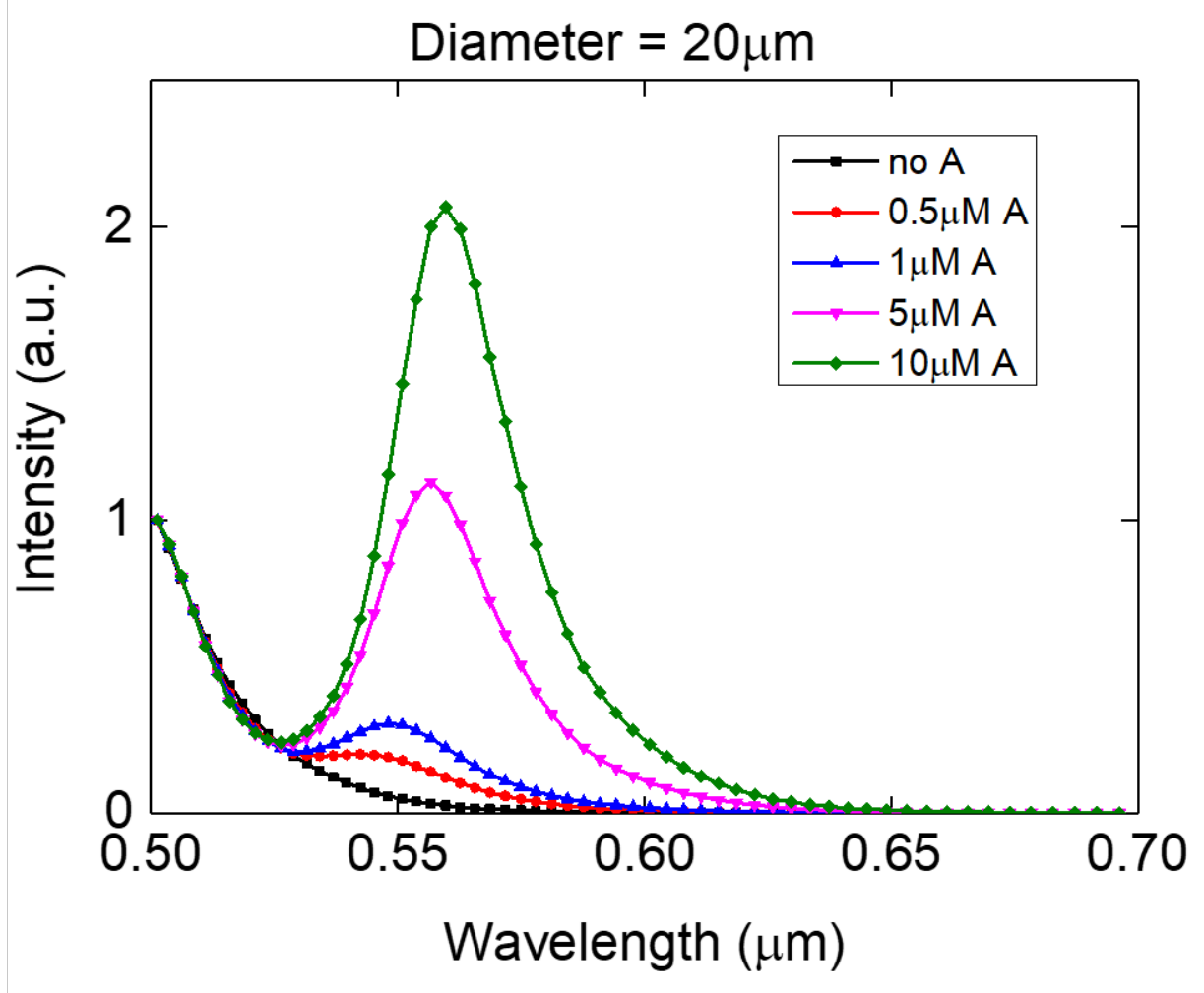

Figure S7. Optical simulation of the intensity of resonant modes (the dots) under different acceptor concentration $(0 \mu \mathrm{M} \sim 10 \mu \mathrm{M})$. The cavity size was fixed as a constant $(\mathrm{D}=20 \mu \mathrm{m})$. 


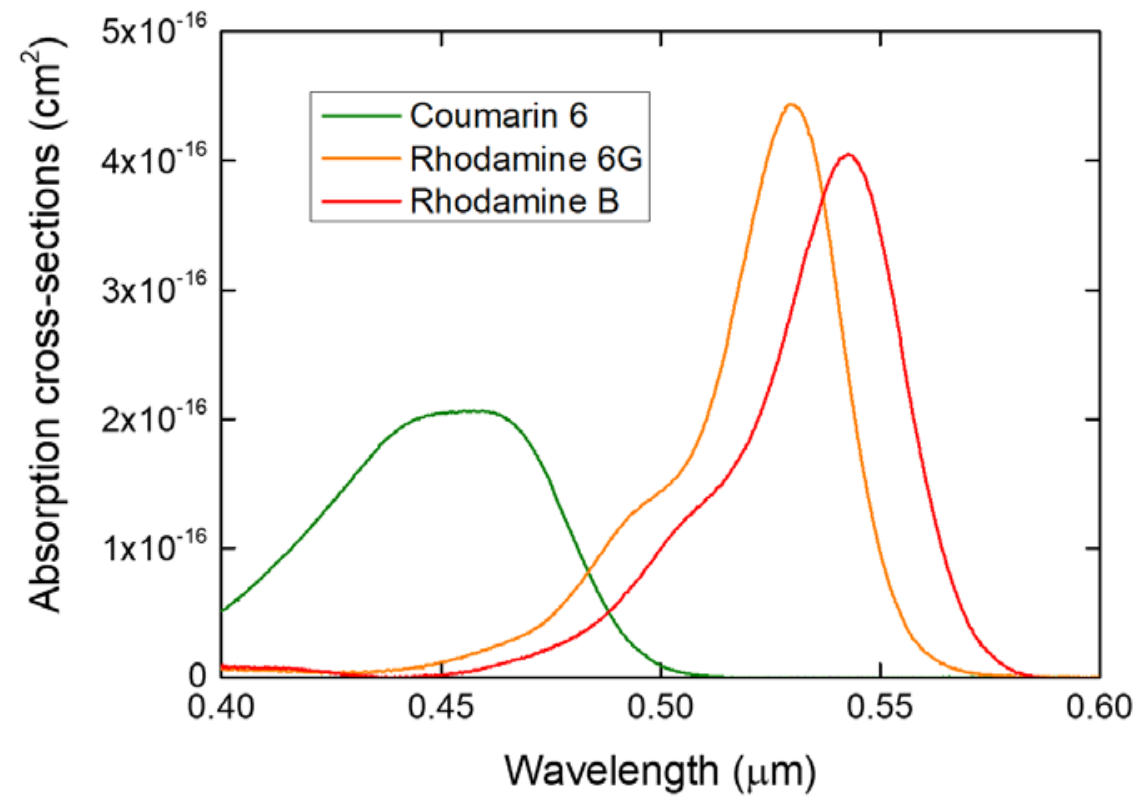

Figure S8. Absorption cross-sections of three fluorescent dyes Coumarin 6 (C6), Rhodamine 6G (R6G), and Rhodamine B (RhB). 


\section{Description of Barcode Complexity Calculation}

As illustrated in Table S1, by ignoring the higher-order modes in both Figures 2a and 2b, the number of remaining fundamental resonant modes (wavelengths) are 40 (with the donor) and 31 (without donor), respectively. Considering that the intensity of each mode is defined by 10 (2) states $(0.1,0.2,0.3,0.4 \ldots 1.0$ or 0,1$)$, then there are respectively $10^{40}\left(2^{40}\right)$ and $10^{31}\left(2^{31}\right)$ combinations for the spectra in Figures 2a and 2b (which is far above trillions). Hence, the barcode will be additional $10^{9}\left(2^{9}\right)$ times "more" complex after introducing the donor to the cavity $\left(10^{40}\right.$ $/ 10^{31}$ or $\left.2^{40} / 2^{31}\right)$. This is how we calculated the case in our paper. However, the number of spectral resonant modes will vary by changing the droplet size and fluorophores (concentrations and bandwidth).

\section{Table S1. Barcode Complexity Calculation}

\begin{tabular}{|c|c|c|c|}
\hline & $\begin{array}{c}\text { Number of } \\
\text { fundamental } \\
\text { modes }\end{array}$ & $\begin{array}{c}\text { The complexity with 10 } \\
\text { states (0.1-1) }\end{array}$ & $\begin{array}{c}\text { The complexity with 2 } \\
\text { states (0 and 1) }\end{array}$ \\
\hline $\begin{array}{c}\text { In presence of the donor } \\
\text { (Fig. 2a) }\end{array}$ & 40 & $10^{40}$ & $2^{40}$ \\
\hline $\begin{array}{c}\text { In absence of the donor } \\
\text { (Fig. 2b) }\end{array}$ & 31 & $10^{31}$ & $2^{9}$ \\
\hline Difference & 9 & $10^{9}$ & \\
\hline
\end{tabular}




\section{Eigenmode Calculation}

In our experiment, the orientation of the liquid crystal molecule is radial, which means the rod-like molecule is perpendicular to the surface of the microdroplet. Typically, nematic liquid crystal (5CB) has high birefringence with ordinary refractive index (RI) $n_{o}=1.54$ (perpendicular to the long axis of NLC molecule) and extraordinary refractive index $n_{e}=1.72$ (parallel to the long axis of NLC molecule). Taking into account the refractive index of the aqueous ambient $\left(n_{s}=n_{H 2 O}=1.33\right)$, the transverse electric $(\mathrm{TE})$ mode suffers a smaller RI $(1.54 / 1.33 \approx 1.16)$ than transverse magnetic $(\mathrm{TM})$ mode $(1.72 / 1.33 \approx 1.29)$, which means TE modes sense more radiation loss. Moreover, the contribution of the material absorption loss to the quality factor is given by $Q_{m a t}=\frac{2 \pi n}{\alpha \lambda}$, indicating that TE mode experience more material absorption loss. In summary, only TM mode but not TE mode can be supported by the NLC microdroplet.

For dielectrically anisotropic sphere (long axis in radial orientation), we have the characteristic equation as follows ${ }^{11}$ :

$$
\begin{gathered}
T E: \frac{n_{o}}{n_{s}} \frac{\left[\kappa_{l, s} R j_{l}\left(\kappa_{l, s} R\right)\right]^{\prime}}{\kappa_{l, s} R j_{l}\left(\kappa_{l, s} R\right)}=\frac{\left[\tilde{\kappa}_{l, s} R h_{l}^{(1)}\left(\tilde{\kappa}_{l, s} R\right)\right]^{\prime}}{\tilde{\kappa}_{l, s} R h_{l}^{(1)}\left(\tilde{\kappa}_{l, s} R\right)} \\
T M: \frac{n_{s}}{n_{o}} \frac{\left[\kappa_{l, s} R j_{\tilde{l}}\left(\kappa_{l, s} R\right)\right]^{\prime}}{\kappa_{l, s} R j_{\tilde{l}}\left(\kappa_{l, s} R\right)}=\frac{\left[\tilde{\kappa}_{l, s} R h_{\tilde{l}}^{(1)}\left(\tilde{\kappa}_{l, s} R\right)\right]^{\prime}}{\tilde{\kappa}_{l, s} R h_{\tilde{l}}^{(1)}\left(\tilde{\kappa}_{l, s} R\right)}
\end{gathered}
$$

where, $\kappa_{l, s}=n_{o} \omega_{l, s} / c, \tilde{\kappa}_{l, s}=n_{s} \omega_{l, s} / c$, and c is the speed of light in vacuum, $\omega_{l, s}=2 \pi f_{l, s}$ is the eigenfrequency with polar mode number $l$ and radial mode number $s \cdot j_{l}$ and $h_{l}^{(1)}$ are spherical Bessel function of the first kind and spherical Hankle function of the first kind,

respectively. $\mathrm{R}$ is the radius of the sphere. $\tilde{l}=\tilde{l}(l)=\frac{1}{2}\left[-1+\sqrt{1+4\left(n_{o} / n_{e}\right)^{2} l(l+1)}\right]$ is a non-integer order.

Subsequently, we fitted the resonance peak as TM modes by anisotropic characteristic equation (eq. S2), including polar mode number $l$ and radial mode number $s$, and the result is reasonable. 


\section{Electric Field Calculation}

When the information $(l, s, \omega)$ of the eigenmodes were obtained by solving eq. S1-S2, electric (magnetic) field can be calculated from the general description of field amplitude (for simplicity, we only presented equations for TM modes) $)^{42}$ :

$$
\begin{aligned}
& E_{r}^{T M}(r, \theta, \varphi)=\left\{\begin{array}{cr}
\tilde{l}(\tilde{l}+1) \frac{j_{\tilde{l}}\left(\kappa_{l, s} r\right)}{\kappa_{l, s} r} Y_{l}^{m}(\theta, \varphi), & r<R \\
\frac{n_{o}}{n_{s}} \frac{j_{\tilde{l}}\left(\kappa_{l, s} R\right)}{h_{\tilde{l}}^{(1)}\left(\tilde{\kappa}_{l, s} R\right)} \tilde{l}(\tilde{l}+1) \frac{h_{\tilde{l}}^{(1)}\left(\tilde{\kappa}_{l, s} r\right)}{\tilde{\kappa}_{l, s} r} Y_{l}^{m}(\theta, \varphi), & r>R
\end{array}\right. \\
& E_{\theta}^{T M}(r, \theta, \varphi)=\left\{\begin{array}{cc}
\frac{\left[\kappa_{l, s} r j_{\tilde{l}}\left(\kappa_{l, s} r\right)\right]^{\prime}}{\kappa_{l, s} r} \frac{\partial Y_{l}^{m}(\theta, \varphi)}{\partial \theta}, & r<R \\
\frac{n_{s}}{n_{o}} \frac{\left[\kappa_{l, s} R j_{\tilde{l}}\left(\kappa_{l, s} R\right)\right]^{\prime}}{\left[\tilde{\kappa}_{l, s} R h_{\tilde{l}}^{(1)}\left(\tilde{\kappa}_{l, s} R\right)\right]^{\prime}} \frac{\left[\tilde{\kappa}_{l, s} r h_{\tilde{l}}^{(1)}\left(\tilde{\kappa}_{l, s} r\right)\right]^{\prime}}{\tilde{\kappa}_{l, s} r} \frac{\partial Y_{l}^{m}(\theta, \varphi)}{\partial \theta}, & r>R
\end{array}\right. \\
& E_{\phi}^{T M}(r, \theta, \varphi)=\left\{\begin{array}{cc}
i \frac{m}{\sin \theta} \frac{\left[\kappa_{l, s} r j_{\tilde{l}}\left(\kappa_{l, s} r\right)\right]^{\prime}}{\kappa_{l, s} r} Y_{l}^{m}(\theta, \varphi), & r<R \\
\frac{n_{s}}{n_{o}} \frac{\left[\kappa_{l, s} R j_{\tilde{l}}\left(\kappa_{l, s} R\right)\right]^{\prime}}{\left[\tilde{\kappa}_{l, s} R h_{\tilde{l}}^{(1)}\left(\tilde{\kappa}_{l, s} R\right)\right]^{\prime}} i \frac{m}{\sin \theta} \frac{\left[\tilde{\kappa}_{l, s} r h_{\tilde{l}}^{(1)}\left(\tilde{\kappa}_{l, s} r\right)\right]^{\prime}}{\tilde{\kappa}_{l, s} r} Y_{l}^{m}(\theta, \varphi), & r>R
\end{array}\right.
\end{aligned}
$$

where $E_{r}^{T M}, E_{\theta}^{T M}$ and $E_{\phi}^{T M}$ represent components of electric field in spherical coordinates, $(r, \theta, \varphi)$ is position vector in spherical coordinates, $Y_{l}^{m}$ is spherical harmonics function, $m$ is azimuthal mode number.

As shown in Fig. S1, distribution of electric field $\left(\left|E_{T M}\right|^{2}=E_{r}^{T M^{2}}+E_{\theta}^{T M^{2}}+E_{\phi}^{T M^{2}}\right)$ along the radial direction was calculated by setting $\theta=\pi / 2$ and $\varphi=0$. The discontinuous at the interface is attributed to the boundary condition of EM field. 


\section{Theoretical Calculation of Cavity Spontaneous Emission Rate in a Microcavity}

Compared to pulsed laser, continuous-wave (CW) light sources possess a much greater width than the fluorescence lifetime ( ns) and its' energy density is much lower than the pulsed laser. As such, gain medium has little chance to achieve population inversion, which means spontaneous emission dominates rather than stimulated emission and thereby the stimulated emission term was neglected. According to Fermi's golden rule, the rate of photon emission can be written as eq. S6 $6^{36,37}$ :

$$
\Gamma_{e m}=\frac{2 \pi}{\hbar} \int_{0}^{\infty}|\langle f|H| i\rangle|^{2} \rho(\omega) L(\omega) d \omega
$$

where $\rho(\omega)$ is the density of states per unit of angular frequency $\omega, L(\omega)$ is the emission line shape, $|\langle f|H| i\rangle|^{2}$ represents the strength of the coupling between initial and final states.

In a vacuum, the density of states is homogeneous so that the rate of photon emission is exactly equal to radiative emission rate of dyes:

$$
\begin{aligned}
\kappa_{\text {rad }}=\Gamma_{\text {em,vac }} & =\frac{2 \pi}{\hbar} \int_{0}^{\infty}|\langle f|H| i\rangle|^{2} \rho_{\text {vac }} L(\omega) d \omega \\
& =\frac{2 \pi}{\hbar}|\langle f|H| i\rangle|^{2} \rho_{\text {vac }}
\end{aligned}
$$

In the presence of the cavity, the density of states at the resonant modes is supposed to be enhanced by Purcell factor:

$$
F_{p}(\omega)=\frac{\rho_{c a v}(\omega)}{\rho_{v a c}}
$$

Considering eq. S6-8, we derived the photon emission rate in the microcavity as follows:

$$
\begin{aligned}
& \Gamma_{\text {em,cav }}=\frac{2 \pi}{\hbar} \int_{0}^{\infty}|\langle f|H| i\rangle|^{2} \rho_{\text {cav }}(\omega) L(\omega) d \omega \\
= & \frac{2 \pi}{\hbar} \int_{0}^{\infty}|\langle f|H| i\rangle|^{2} F_{p}(\omega) \rho_{v a c} L(\omega) d \omega \\
= & \kappa_{\text {rad }} \int_{0}^{\infty} F_{p}(\omega) L(\omega) d \omega
\end{aligned}
$$




\section{FRET rate}

Förster resonant energy transfer is a near-field electrodynamic phenomenon that has been explained by both classical physics and quantum physics. The FRET rate can be written as $^{43}$ :

$$
\kappa_{F}=\frac{1}{\tau_{D}}\left(\frac{R_{0}}{r}\right)^{6}
$$

where $\tau_{D}$ is the intrinsic fluorescence lifetime of the donor, $r$ is the distance between donor and acceptor molecule, $R_{0}$ is the Förster distance given by ${ }^{43}$ :

$$
R_{0}=\sqrt[6]{\frac{9 \ln (10) \kappa^{2} \phi_{D}}{128 \pi^{5} N_{A} n_{S}^{4}} \int_{0}^{\infty} F_{D}(\lambda) \epsilon_{A}(\lambda) \lambda^{4} d \lambda}
$$

Here, $\phi_{D}$ is the quantum yield of the donor, $\kappa^{2}$ is the orientation factor and usually assumed to be $2 / 3, N_{A}$ is the Avogadro's number, $n_{s}$ is the refractive index of the surrounding medium, $F_{D}(\lambda)$ and $\epsilon_{A}(\lambda)$ represent the area-normalized donor emission intensity and extinction coefficient of the acceptor $\left(\mathrm{M}^{-1} \mathrm{~cm}^{-1}\right)$, respectively.

We selected Coumarin 6 as donor and Rhodamine 6G as acceptor, and the calculated Förster distance is $6.08 \mathrm{~nm}$ in water and $5.51 \mathrm{~nm}$ in liquid crystal. Inside the microcavity, the average distance between donor molecules can be estimated by $r_{a v g}=1 / \sqrt[3]{N_{A} C}$ and the D-A distance $r$ at the interface is supposed to be less than $r_{a v g}$. 
Table S2. Simulation parameters

\begin{tabular}{|c|c|c|}
\hline Symbols & Description & Numeric value \\
\hline$n_{o}$ & The ordinary refractive index of 5CB & 1.54 \\
\hline$n_{e}$ & The extraordinary refractive index of 5CB & 1.72 \\
\hline$n_{s}$ & The refractive index of the aqueous ambient & 1.33 \\
\hline$\sigma_{a b s, D}$ & Absorption cross-section of donor & See Fig. S8 \\
\hline$\sigma_{a b s, A}$ & Absorption cross-section of acceptor & See Fig. S8 \\
\hline$L_{D}$ & Emission probability of donor & See Fig. S3 \\
\hline$L_{A}$ & Emission probability of acceptor & See Fig. S3 \\
\hline$I_{p h}$ & The power density of the pump & $1.33 \mathrm{~mW} / \mathrm{cm}^{2}$ \\
\hline$c$ & Speed of light in vacuum & $30 \mathrm{~cm} / \mathrm{ns}$ \\
\hline$h$ & Plank constant & $6.626 \mathrm{e}-34 \mathrm{~J} \cdot \mathrm{s}$ \\
\hline$N_{A}$ & Avogadro constant & $6.022 \mathrm{e} 23 \mathrm{~mol}^{-1}$ \\
\hline$\tau_{D}$ & Donor intrinsic fluorescence lifetime & C6: $2.5 \mathrm{~ns}$ \\
\hline$\tau_{A}$ & Acceptor intrinsic fluorescence lifetime & R6G: 4.08 ns RhB: $1.68 \mathrm{~ns}$ \\
\hline$\phi_{D}$ & Donor quantum yield & 0.78 \\
\hline$\phi_{A}$ & Acceptor quantum yield & R6G: 0.95 RhB: 0.65 \\
\hline$\kappa^{2}$ & orientation factor & $2 / 3$ \\
\hline
\end{tabular}

